\title{
A taxa de câmbio como instrumento do desenvolvimento econômico: um diálogo com professor Rodolfo Hoffmann
}

\author{
The exchange rate as an instrument of economic development: \\ a dialogue with Professor Rodolfo Hoffmann
}

\author{
LAURO MATTEI* \\ GUILHERME DE OLIVEIRA** \\ THAÍS SCARAMUZZI $* * *$
}

\section{INTRODUÇÃO}

No volume 36, número 4 (145), de outubro-dezembro de 2016, da Revista de Economia Política, escrevemos o artigo "A taxa de câmbio como instrumento do desenvolvimento econômico", procurando mostrar a importância dessa política no desenvolvimento de cada país (Mattei e Scaramuzzi, 2016).

O artigo apresentou um estudo empírico sobre a relação entre câmbio e comércio externo com objetivo de subsidiar o argumento central e as conclusões obtidas por diversos autores citados no trabalho (Bresser-Pereira, 2012; Marçal e Holland, 2011; Margarido, Serigati e Perosa, 2011; Marconi e Barbi, 2011). Para tanto, elaborou-se um modelo econométrico que buscava estimar o impacto marginal da taxa de câmbio sobre a pauta exportadora e importadora do país, classificada de acordo com seus níveis de intensidade tecnológica.

Ficamos extremamente agradecidos pela leitura atenta e crítica feita pelo Professor Hoffmann, cujo teor também faz parte do presente número da Revista de Economia Política. Tal crítica se restringe à analise econométrica do artigo e pode ser sintetizada nos seguintes pontos centrais: a) No modelo em questão, é inapropriado estimar um modelo sem intercepto; b) A interpretação dos testes econométricos em algumas passagens não é clara; c) Há certa redundância em ajustar as três regressões, uma vez que as três participações nas exportações somam sempre 1 ; d) O baixo número de observações torna mais difícil que qualquer fenômeno es-

\footnotetext{
* Professor do curso de Graduação em Economia e do Programa de Pós-Graduação em Administração, ambos da UFSC. Email: 1.mattei@ufsc.br

** Professor do curso de Graduação em Economia da UFSC. Email: oliveira.guilherme@ufsc.br

*** Mestre em Economia pela EESP-FGV. Email: scaramuzzi@gmail.com
} 
tatístico seja captado. Hoffmann finaliza suas críticas deixando duas indagações: $\left.1^{a}\right)$ Não seria melhor considerar o valor exportado ou importado, em lugar de sua participação no total?; $2^{a}$ ) Não seria importante incluir outras variáveis explanatórias?

Levando em consideração as críticas que, em sua maioria, julgamos procedentes, estamos apresentando uma reanálise do assunto com o objetivo de tentar responder, minimamente, aos questionamentos deixados pelo Professor Hoffmann.

\section{ESTRATÉGIA DE IDENTIFICAÇÃO}

Nessa versão, focamos em três pontos da crítica do Professor Hoffman, tratando de maneira implícita pontos menores igualmente relevantes. Inicialmente consideramos a questão do tamanho da amostra. Mesmo num contexto de mínimos quadrados ordinários, uma amostra escassa impossibilita que as propriedades assintóticas do estimador sejam evocadas. Ademais, torna-se mais difícil observar qualquer fenômeno estatístico, como a presença de heterocedasticidade, por exemplo. Para contornar essa limitação, expandimos a amostra para 80 observações, trabalhando com dados trimestrais entre 1997 e 2016.

Posteriormente, incluímos como variável dependente o valor das exportações e das importações por conteúdo tecnológico. Essa nova estratégia, além de enfrentar as questões relacionadas à forma funcional e à identificação dos parâmetros, permite que sejam incorporadas outras variáveis explicativas no modelo, acompanhadas de uma justificativa teórica. O impacto marginal computado sobre o valor não impede que sejam analisadas implicações para a participação de cada segmento na pauta do comércio exterior.

Finalmente, recorremos à estimação de funções de exportações e importações em sua forma reduzida, uma estratégia de identificação amplamente difundida na literatura (Schettini, Squeff e Gouvêa, 2012). Tais equações foram estimadas separadamente. Nesse sentido, apesar de suspeitarmos que os erros padrões estejam relacionados, a estimação individual, apesar de afetar as estatísticas dos testes de hipóteses, não deve causar viés na identificação dos coeficientes associados ao efeito da taxa real de câmbio.

É importante registrar que a presente estratégia de identificação é um dos vários caminhos possíveis de estimação do efeito da taxa de câmbio sobre o comércio internacional. Existem razões, por exemplo, para acreditar que eventuais mudanças de regime possam afetar os resultados, o que sugere a existência de equilíbrios múltiplos na relação entre câmbio, exportações e importações. Contudo, a presente estratégia manteve-se relativamente fiel à proposta inicial do artigo, base da crítica do Professor Hoffmann.

Motivados por essas considerações, estimamos a seguinte forma funcional para a função de exportações brasileira no período entre 1996 e 2016:

$$
X_{i t}=\beta_{0}+\beta_{1} Y_{t}^{m}+\beta_{2} R E R_{t}+\beta_{3} D R C+\beta_{j} D T_{j}+\mu_{t}
$$


em que:

$X_{i t}=$ representa o logaritmo do valor das exportações em reais de 2010. O subscrito $i$ não indica painel, mas sim a classificação por intensidade tecnológica da pauta exportadora estimada individualmente: $X_{0 t}$ representa o valor das exportações sem conteúdo tecnológico; $X_{1 t}$ o conteúdo de baixa e média-baixa intensidade tecnológica; e $X_{2 t}$ representa o valor das exportações de média-alta e alta intensidade tecnológica. Os dados foram obtidos junto ao MDIC (2017);

$Y_{t}^{m}=$ representa a renda mundial. Como proxy para a renda mundial foi utilizado o logaritmo do valor das importações do resto do mundo deflacionado pelo valor unitário das importações mundiais, em dólares de 2010. A série e o deflator foram obtidos na base International Financial Statistics (IFS) do Fundo Monetário Internacional (FMI, 2017);

$R E R_{t}=$ representa o logaritmo da taxa de câmbio real, computada pela taxa nominal de câmbio multiplicada pela razão entre o índice de preços americano e brasileiro. A taxa nominal de câmbio foi coletada no banco de dados IPEADATA (IPEA, 2017) e os índices de preço foram obtidos no IFS (FMI, 2017);

$D R C$ = é uma variável dicotômica que assume 1 para o período de câmbio flutuante e 0 no caso contrário;

$D T$ = é um conjunto de variáveis dummies trimestrais, com $j$ representando o primeiro, segundo e o terceiro trimestres, respectivamente.

$\mu_{t}=$ é o termo de perturbação estocástico; e

$\beta_{i}=$ são os coeficientes a serem estimados.

Já para a função importações supõe-se a seguinte forma funcional:

$$
M_{i t}=\alpha_{0}+\alpha_{1} Y_{t}+\alpha_{2} R E R_{t}+\alpha_{3} D R C+\alpha_{j} D T_{j}+e_{t}
$$

em que:

$M_{i t}=$ representa o logaritmo do valor das importações em reais de 2010 . O subscrito $i$ não indicada painel, mas sim a classificação por intensidade tecnológica da pauta de importações: $M_{0 t}$ representa o valor das importações sem conteúdo tecnológico; $M_{1 t}$ o conteúdo de baixa e média-baixa intensidade tecnológica; e $M_{2 t}$ representa o valor das importações de média-alta e alta intensidade tecnológica. Os dados foram obtidos no MDIC (2017);

$Y_{t}=$ representa a renda interna, medida por meio do logaritmo valor do produto interno bruto em reais de 2010. A fonte de dados foi o Banco de Dados Agregados do SIDRA (IBGE, 2017);

$e_{t}=$ é o termo de perturbação estocástico; e

$\alpha_{i}=$ são os coeficientes a serem estimados.

É importante destacar que mantivemos a classificação das exportações e importações conforme seu conteúdo tecnológico utilizada na versão anterior do artigo. Assim, somaram-se as categorias baixa e média-baixa, bem como as de média-alta e alta intensidade tecnológica.

Todas as variáveis quantitativas foram utilizadas em escala logarítmica com a finalidade de computar diretamente as elasticidades. Os valores nominais foram 
deflacionados pelo índice de preços brasileiro, exceto o valor das importações, cujo deflator foi o valor unitário das importações em dólares. Ambas as séries foram coletadas no IFS do FMI (2017).

A Figura 1 apresenta a evolução temporal das séries utilizadas no modelo, ainda sem a transformação do logaritmo. Nota-se que os valores das importações e exportações elevaram-se no período, mas o padrão de especialização no comércio internacional brasileiro é latente: exportar produtos de baixa ou nenhum intensidade tecnológica e importar produtos de média-alta e alta intensidade tecnológica. Também é possível observar o padrão sazonal das séries, o que exigiu o tratamento apropriado das mesmas. Do mesmo modo, observou-se que a taxa de câmbio real também registrou períodos de apreciação e depreciação ao longo do intervalo analisado.

Figura 1: Evolução do valor das exportações, importações, renda interna, importações do resto do mundo e taxa real de câmbio no Brasil entre 1997 e 2016
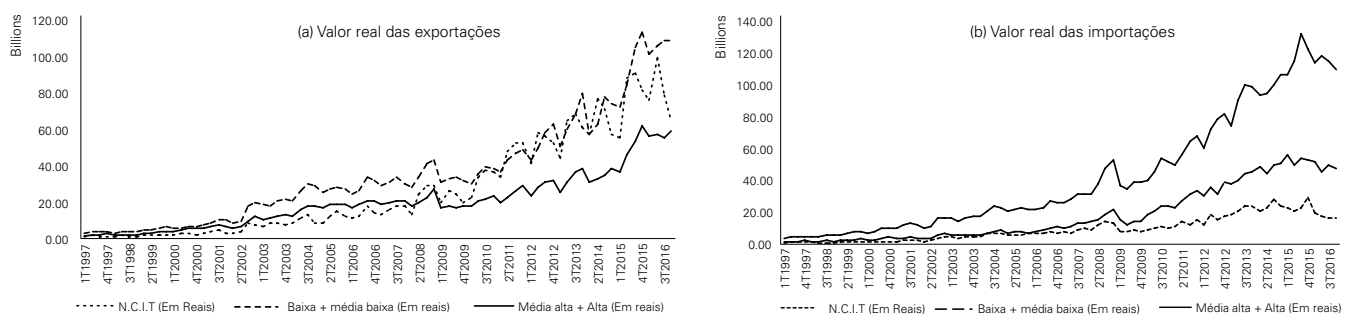

(c) Taxa real de câmbio R / US\$
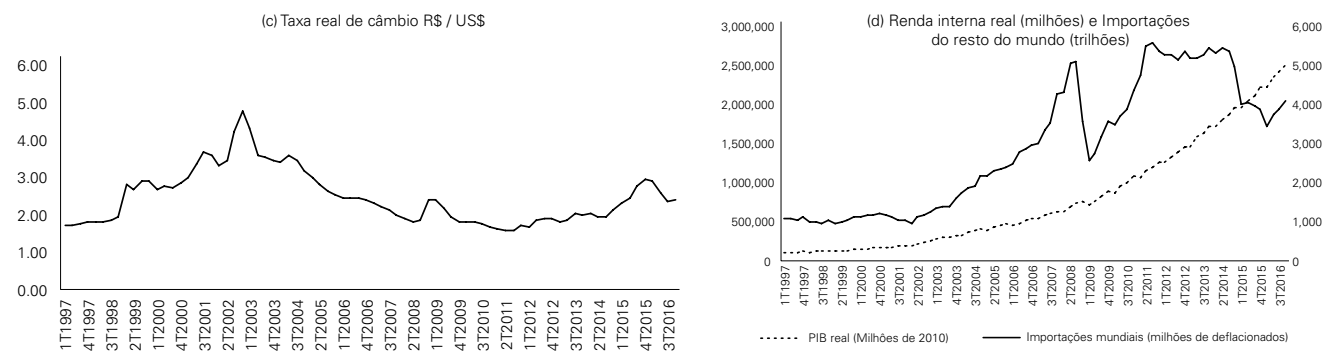

De posse das séries transformadas aplicamos o teste Aumentado de Dickey-Fuller (ADF) para detecção de raiz unitária. Para níveis de 1\% e 5\% de significância ambas as séries foram consideradas integradas de ordem 1 . Nesse contexto, a propriedade de estacionariedade da série, necessária para obtenção de estimativas confiáveis por meio de mínimos quadrados ordinários, não é mais atendida. Optou-se pela estimação por meio da técnica de vetores autorregressivos (VAR) que, dentre outas características, minimiza eventuais problemas de endogeneidade das formas funcionais das funções exportações e importações.

Em seguida, procuramos relações de longo prazo entre as variáveis, por meio da análise de cointegração. O procedimento de Johansen foi aplicado às especifi- 
cações (1) e (2), em um modelo autorregressivo. Foram rodados 12 VARs ao testar as três classificações de intensidade tecnológica com e sem o controle exógeno das dummies trimestrais. Em ambos os casos, definiu-se a defasagem ótima do modelo e aplicou-se o procedimento de Johansen, avaliado por meio da estatística Trace, máximo autovalor e dos critérios de informação. Foram encontradas evidências de cointegração em 10 dos 12 modelos, que apontaram 1 vetor de cointegração nas especificações mencionadas. A exceção foi a função de importações de produtos de baixa e a de alta intensidade tecnológica.

Constatada a existência de cointegração entre as variáveis, utilizamos o mecanismo tradicional de correção de erros para estimar um VEC. Como mencionado, testaram-se especificações com e sem o controle exógeno das dummies trimestrais, porém as versões estimadas sem as dummies apresentaram autocorrelação nos resíduos. As demais hipóteses, relacionadas à normalidade dos resíduos, ausência de autocorrelação e estabilidade das raízes da equação característica, foram atendidas em todos os modelos que incluem as dummies, o que demonstra sua relevância na especificação testada.

\section{Resultados}

Em um contexto de desenvolvimento econômico, a análise relevante é a de longo prazo. Portanto, nessa exposição o foco se dá sobre as relações de longo prazo expressas pela equação cointegrante do VEC. Os mecanismos de ajustamento e os coeficientes do VAR, cujo foco reside no curto prazo, não são discutidos.

A Tabela 1 resume os resultados encontrados para a função de exportações. Nas colunas encontram-se as variáveis dependentes separadas pela classificação do MDIC de intensidade tecnológica, com mencionado anteriormente. Nas linhas estão os regressores. A definição se o modelo inclui ou não a constante e a tendência linear foi definida no procedimento de Johansen, seguindo a minimização dos critérios de informação. Por essa razão, por exemplo, a forma funcional estimada para a variável exportação de média-alta e alta tecnologia com variáveis dummies não inclui intercepto e tendência linear.

Nota-se que no modelo com dummies, a elasticidade das exportações com relação à renda do resto do mundo foi estatisticamente significativa. O impacto marginal de uma elevação na proxy da renda externa é mais forte sobre a exportação de produtos sem conteúdo tecnológico, como bens agrícolas, por exemplo. Uma elevação de $1 \%$ na renda externa eleva as exportações de produtos não tecnológicos em 3,62\%. Por sua vez, a elasticidade-renda das exportações de produtos de média-alta e alta intensidade tecnológica foi de $2,61 \%$, enquanto que a elasticidade-renda das exportações de produtos de baixa e média baixa intensidade tecnológica foi de $2,75 \%$. A identificação dos parâmetros é prejudicada no modelo sem controle da sazonalidade trimestral, o que resulta na instabilidade dos coeficientes. Entretanto, o sinal é sempre positivo, de acordo com o esperado pela teoria econômica.

As elasticidades associadas à taxa de câmbio real foram positivas e estatistica- 
mente significativas no modelo que inclui as dummies trimestrais. Uma depreciação do real em relação ao dólar expande relativamente mais a exportação de bens de baixa e média-baixa intensidade tecnológica, 4,25\%. Estaticamente, tal elasticidade é equivalente à associada aos produtos sem conteúdo tecnológico, 4,22\%. Por outro lado, uma depreciação de $1 \%$ na moeda nacional expande as exportações classificadas como de média-alta e alta tecnologia em 3,74\%. A exclusão dos controles trimestrais mais uma vez se mostrou inadequada.

Tabela 1: Equação cointegrante e relações de longo prazo na função de exportações brasileira entre 1996 e $2016^{*}$

\begin{tabular}{|c|c|c|c|c|c|c|}
\hline \multirow[t]{2}{*}{ Regressores } & \multicolumn{6}{|c|}{ Variáveis dependentes } \\
\hline & $x_{0}$ & $X_{1} * *$ & $X_{2}^{* *}$ & $x_{0}$ & $x_{1}$ & $x_{2}$ \\
\hline \multicolumn{7}{|c|}{ Equação cointegrante e relações de longo prazo } \\
\hline \multirow[t]{2}{*}{ C } & -8.1342 & - & - & -5.181731 & 21.84567 & $20.65519^{* * *}$ \\
\hline & {$[-2.98934]$} & - & - & {$[-2.62409]$} & {$[6.96327]$} & - \\
\hline \multirow[t]{2}{*}{$Y^{m}(-1)$} & 3.617721 & 2.749845 & 2.674371 & 3.404205 & 0.359783 & 0.059748 \\
\hline & {$[10.7656]$} & {$[58.0625]$} & {$[48.0640]$} & {$[14.1029]$} & {$[0.95178]$} & {$[0.19275]$} \\
\hline \multirow[t]{2}{*}{$R E R(-1)$} & 4.220835 & 4.253789 & 3.74041 & 3.243535 & -3.495725 & 0.738923 \\
\hline & [5.62557] & [14.3978] & {$[10.6636]$} & [5.98211] & {$[-3.52918]$} & [2.88950] \\
\hline Tendência & - & - & - & - & - & 0.039743 \\
\hline \multicolumn{7}{|c|}{ Controles exógenos do VAR } \\
\hline $\begin{array}{l}\text { Dummy de regime } \\
\text { cambial }\end{array}$ & Sim & Sim & Sim & Sim & Sim & Sim \\
\hline $\begin{array}{l}\text { Dummies } \\
\text { trimestrais }\end{array}$ & Sim & Sim & Sim & Não & Não & Não \\
\hline R2_Ajustado & 0.611888 & 0.621112 & 0.599795 & 0.607783 & 0.461443 & 0.582898 \\
\hline
\end{tabular}

* Estatística t entre colchetes. ** Modelo sem constante e tendência linear. *** No modelo com tendência, o Eviews 9.0 não reporta as estatísticas de teste para a constante.

Apesar do sinal da elasticidade associada à taxa real de câmbio continuar positivo e estatisticamente significativo, ao contrário do encontrado no modelo estimado por mínimos quadrados ordinários do artigo anterior, seu valor é relativamente mais baixo no segmento das exportações de produtos de média-alta e alta tecnologia. Nos três casos, entretanto, a qualidade do ajuste, medida pelo $\mathrm{R}^{2}$ ajustado, mostrou-se satisfatória, em face da quantidade de variáveis independentes. A escolha do $\mathrm{R}^{2}$ ajustado ao invés do $\mathrm{R}^{2}$ se dá pela punição que o primeiro concede à inclusão de mais variáveis explicativas no modelo.

Portanto, as evidências encontradas sugerem que a taxa real de câmbio exerce um papel pertinente no longo prazo para a expansão das exportações em todos os seus níveis de intensidade tecnológica. Além disso, sugere também que a taxa real de câmbio não é uma condição suficiente para a uma reconversão do padrão de especialização no comércio internacional do Brasil, já que o seu impacto marginal 
é relativamente mais fraco sobre as exportações de produtos de média-alta e alta intensidade tecnológica.

Quanto à função de importações, o modelo empírico se mostrou menos satisfatório. A qualidade do ajuste da função de importações é relativamente baixa, mesmo no modelo que inclui os controles sazonais. $\mathrm{O} \mathrm{R}^{2}$ ajustado observado é relativamente mais baixo, ao passo que a não inclusão dos controles exógenos eleva a instabilidade das elasticidades estimadas.

A Tabela 2 apresenta uma interpretação similar à Tabela 1 . O modelo que incluiu as variáveis dummies como controles exógenos também se mostrou o mais adequado. Mesmo assim, o impacto marginal que a renda interna exerceu sobre a demanda de importações de produtos sem conteúdo tecnológico, ou de baixa e baixa-média intensidade tecnológica, não se mostrou estaticamente significativo, apesar do sinal ser corretamente captado.

O efeito que a renda interna exerce sobre as importações de produtos de média-alta e alta tecnologia se mostrou significativo ao nível de $10 \%$, indicando que uma elevação de $1 \%$ no PIB eleva as importações em $0,65 \%$. Ademais, como mostra a Tabela 2, em termos absolutos, a elasticidade-renda das importações é maior nos produtos de elevado conteúdo tecnológico.

Não foram encontradas evidências de que a taxa real de câmbio exerceu influência sobre a demanda de importação de produtos de média-alta e alta intensidade tecnológica. As estimativas indicam que a elasticidade das importações é mais forte para produtos de baixa-média e baixa intensidade tecnológica e, especialmente, nos bens sem conteúdo tecnológico. No primeiro caso, uma depreciação cambial de $1 \%$ reduz a as importações $0,3919 \%$, enquanto na última categoria a redução é de $18,24 \%$.

Tabela 2: Equação cointegrante e relações de longo prazo na função de importações brasileira entre 1996 e 2016.*

\begin{tabular}{|c|c|c|c|c|}
\hline \multirow{2}{*}{ Regressores } & \multicolumn{4}{|c|}{ Variáveis dependentes } \\
\hline & Mo & $M_{1}$ & $M_{2}$ & Mo \\
\hline \multicolumn{5}{|c|}{ Equação cointegrante e relações de longo prazo } \\
\hline \multirow[t]{2}{*}{ C } & -33.00935 & $17.31916 * *$ & $14.71434 * *$ & 11.33166 \\
\hline & [ 0.77825] & - & - & [3.6123] \\
\hline \multirow[t]{2}{*}{$Y(-1)$} & 0.561452 & 0.376435 & 0.665444 & 0.862989 \\
\hline & [0.17554] & [0.91585] & [1.83206] & [10.0648] \\
\hline \multirow[t]{2}{*}{ RER (-1) } & -18.2425 & -0.39191 & -0.039556 & -0.136508 \\
\hline & {$[-1.65504]$} & [-3.75498] & {$[-0.42795]$} & [ - 0.41864$]$ \\
\hline Tendência & - & 0.029291 & 0.014709 & - \\
\hline
\end{tabular}


Controles exógenos do VAR

\begin{tabular}{lcccc}
\hline Dummy de regime cambial & Sim & Sim & Sim & Sim \\
Dummies trimestrais & $\operatorname{Sim}$ & $\operatorname{Sim}$ & $\operatorname{Sim}$ & Não \\
\hline R2_Ajustado & 0.190143 & 0.409665 & 0.571975 & 0.157803 \\
\hline
\end{tabular}

* Não foram encontradas evidências de cointegração e, portanto, equilíbrio de longo prazo para as variáveis M1 e M2. ** No modelo com tendência, o Eviews 9.0 não reporta as estatísticas de teste para a constante.

É interessante destacar que a fração das importações brasileiras com produtos classificados como sem conteúdo tecnológico é a mais baixa dos três níveis. Mesmo assim, o efeito marginal associado a uma depreciação do câmbio é o mais elevado. Paralelamente, a importação de produtos com níveis médio-alto e alto nível tecnológico domina a pauta de importações, mas mesmo sem evidências de que a taxa real de câmbio possa exercer algum efeito sobre essas importações, sua elasticidade é relativamente baixa $-0,039 \%$.

\section{CONCLUSÃO}

A crítica do Professor Hoffmann trouxe importantes questões para nossa análise da relação entre a taxa real de câmbio e o comércio exterior no Brasil. Por um lado, a ampliação da amostra permitiu a estimação de parâmetros estáveis e a realização de estatísticas de teste mais eficientes. Por outro lado, a utilização dos valores reais das exportações e importações favoreceu a estimação dessas funções em sua forma reduzida com a inclusão de outras variáveis no modelo, justificadas economicamente por relações teóricas tradicionais.

A utilização de um VEC trouxe novas evidências que subsidiam a literatura em torno da estimação das elasticidades das funções exportação e importação em relação à taxa real de câmbio. Como encontrado no artigo anterior, as elasticidades foram estatisticamente significativas e, em especial, uma elevação da taxa real de câmbio tende a gerar um impacto mais que proporcional sobre o valor real das exportações.

Contudo, os resultados da presente versão vêm com uma importante ressalva: uma depreciação da taxa real de câmbio, apesar de pertinente para a expansão das exportações em todos os seus níveis de intensidade tecnológica, não parece ser uma condição suficiente para a reconversão do atual padrão de especialização do comércio internacional brasileiro, já que o impacto marginal é relativamente mais fraco sobre as exportações de produtos de média-alta e alta intensidade tecnológica.

Se a exportação de bens relativamente mais intensivos em tecnologia é um elemento importante para uma estratégia de desenvolvimento econômico, uma eventual depreciação cambial precisa ser acompanhada de outro conjunto de in- 
centivos econômicos. Martins-Neto e Lima (2016), por exemplo, ressaltam o papel que a coordenação de investimentos públicos em infraestrutura pode ter para potencializar os efeitos da taxa real de câmbio sobre o crescimento econômico.

O presente resultado depende, entretanto, de uma série de hipóteses relacionadas ao próprio modelo econométrico, em especial, da suposição de não alteração de regimes ao longo do tempo e da hipótese de cointegração linear entre as variáveis. A elaboração de novos estudos empíricos que incorporem tais questões pode trazer novas evidências relacionadas ao papel da taxa de câmbio no desenvolvimento econômico.

\section{REFERÊNCIAS BIBLIOGRÁFICAS}

Araújo, E. C. (2009) Nível do câmbio e crescimento econômico: teorias e evidências para países em desenvolvimento e emergentes - 1980 - 2007. Brasília: Ipea.

Bresser-Pereira, L. C. (2012) "A taxa de câmbio no centro da teoria do desenvolvimento". Estudos Avançados, 26 (75): 7-28.

Hoffmann, R. (2017) Comentários sobre o artigo "A taxa de câmbio como instrumento do desenvolvimento econômico". São Paulo, fevereiro de 2017.

International, Monetary Fund (FMI). International Financial Statistics. Disponível em: www.imf.org Acesso em 12 mai. 2014 e 18-19 de julho de 2017.

Instituto Brasileiro de Geografia e Estatística (IBGE). Banco de Dados Agregado (SIDRA). Disponível em: www.ibge.gov.br. Acesso em 12 mai. 2014 e 18-19 de julho de 2017.

Instituto de Pesquisa Econômica Aplicada (IPEA). Ipeadata. Disponível em: <www.ipeadata.gov.br>. Acesso em 12 mai. 2014 e 18-19 de julho de 2017.

Marçal, E.; Holland, M. (2011) “Exportações brasileiras e o papel da taxa de câmbio". In: HOLLAND, Márcio; NAKANO, Yoshiaki (Org.). Taxa de câmbio no Brasil: Estudos de uma perspectiva do desenvolvimento econômico. São Paulo: Campus.

Marconi, N.; Barbi, F. (2011) “Taxa de câmbio e composição setorial da produção”. In: HOLLAND, M.; NAKANO, Y. (Org.). Taxa de câmbio no Brasil: Estudos de uma perspectiva do desenvolvimento econômico. São Paulo: Campus

Margarido, M.A.; Serigatti, F.; Perosa, B. (2011) "Preços internacionais de commodities e a taxa de câmbio real no Brasil”. In: Holland, M.; Nakano, Y. (Org.). Taxa de câmbio no Brasil: Estudos de uma perspectiva do desenvolvimento econômico. São Paulo: Campus.

Martins-Neto, A. S.; Lima, G. T. (2016) "Competitive exchange rate and public infrastructure in a macrodynamic of economic growth". Metroeconomica, p. 1-24, Julho, 2016.

Mattei, L.; Scaramuzzi, T. (2016) "A taxa de câmbio como instrumento do desenvolvimento econômico". Revista de Economia Política, vol.36, n.4 (145), p.726-747.

Ministério do Desenvolvimento, Indústria e Comércio (MDIC) Estatísticas de Comércio Exterior. Disponível em: www.desenvolvimento.gov.br>. Acesso em 18-19 de julho de 2017.

Missio, F.J. (2012) “Câmbio real e mudança estrutural: perspectivas para o Brasil”. In: Modenesi, A. et al., org. Sistema financeiro e politica econômica em uma era de instabilidade: tendências mundiais e perspectivas para a economia brasileira. Rio de Janeiro: Elsevier; São Paulo: AKB.

Schettini, B. P.; Squeff, G. C.; Gouvêa, R. R. (2012) “Estimativas da função exportações brasileiras agregadas com dados das contas nacionais trimestrais, 1995-2009”. Economia Aplicada, v. 16, n.1, 2012. 